\title{
Fungal Infections Masquerading as Gastrointestinal Tract Malignancies - A Series of Three Cases
}

\author{
Chaturvedi Rachana A*, Baste Balaji D, Karegar Manjusha M, Gupte Prajakta A and Amita Joshi S \\ Department of Pathology, Seth G.S. Medical College \& K.E.M. Hospital, Mumbai, Maharashtra
}

\begin{abstract}
Fungal infections of the gastrointestinal tract (GIT) are not rare, but get missed due to their misleading clinical features. Here we report three GIT fungal infections at different locations with varied symptoms. We received surgical resection specimens with the clinical suspicion of malignancy for all. The first was a case of Aspergillosis, at an uncommon site, esophageal, in a 60year diabetic male with a suspicion of carcinoma in view of presence of dysphagia and a stricture on CT. The second was a 60-year male, a case of stomach Zygomycosis with no definite immunosuppression, who presented with an intra-operative suspicion of malignancy, and showed fungal angio-invasion and ischemic perforation. Third was a 32year immunocompetent male, a case of GI histoplasmosis with an uncommon presentation, showing obstructive symptoms due to an ileocaecal mass with a clinical suspicion of TB/malignancy. Thus, a high index of suspicion for fungal a etiology in GIT is essential, because misdiagnosis can lead to a radical surgery. Timely treatment with appropriate antifungal therapy is crucial as high mortality is associated with certain fungi.
\end{abstract}

Keywords: Gastrointestinal Tract, Fungal Infections, Malignancy, Aspergillosis, Zygomycosis, Histoplasmosis

\section{Introduction}

Infections of gastrointestinal (GI) tract are common, usually treatable, and can be caused by various bacteria, viruses, or sometimes fungi. Often the diagnosis is easy due to the characteristic symptoms in association with appropriate investigations. However, clinical features can sometimes be non-classical and overlapping, and these are the situations where simple infections can cause diagnostic difficulties, occasionally can even mimic malignancy. ${ }^{[1]}$

GI tract fungal infections, though preventable and curable, they account for a sizable proportion of cases causing substantial mortality and morbidity in the developing countries. ${ }^{[2]}$ These infections occur mainly in immunocompromised patients, but virtually all have been described in immunocompetent persons as well. Common GI symptoms include diarrhea, vomiting, melena, hemorrhage, abdominal pain, and fever ${ }^{[3]}$ The presentation is often similar regardless of the type of fungus involved. ${ }^{[1]}$

Common GI tract fungal infections include Candida, Aspergillus, Zygomycetes, Histoplasma, and Cryptococcus. GI Aspergillosis (GIA) occurs almost exclusively in immunocompromised patients, with coexistent lung lesions in most. The typical hyphae of Aspergillus are septate with acute angle branching. [3] Gastrointestinal Zygomycosis (GIZ) is the rarest manifestation of mucormycosis accounting for $4-7 \%$ of all cases. In the past, most cases have been reported in adults, mostly with some degree of immuno suppression.
However, in recent years, GIZ have also been reported in patients without underlying traditional risk factors. ${ }^{[4]}$ In contrast to Aspergillus, these organisms have broad, ribbon like, pauci-septate hyphae that branch randomly at various angles. ${ }^{[3]}$ Patients with Gastrointestinal Histoplasmosis (GIH) are usually asymptomatic, and diagnosis is often made incidentally or at autopsy. GIH has been reported, both in immunocompetent and immunocompromised individuals, disseminated forms being more common in the latter group. GIH is common in patients with primary lung involvement, but it is very unusual to present with isolated involvement of the gastrointestinal system. The majority $(90 \%)$ of lesions involve the lower GI tract, most commonly the ileocecal region or colon, likely due to extensive gutassociated lymphoid tissue (GALT) present in this region. These areas serve as potential entry sites for the organisms, which are-small, ovoid, usually intracellular yeast forms in macrophages, with small buds at the more pointed pole. ${ }^{5,}$ ${ }^{3]}$ Appropriate treatment with antifungal therapy definitely improves outcomes; nevertheless, zygomycosis and aspergillosis cases still have high mortality rates. ${ }^{6]}$

Here we report three different fungal infections, at various GI sites, presenting as malignancies.

\section{Case Report}

Case 1: A60-year-old man, a known case of diabetes mellitus presented with a history of dysphagia. His endoscopy and CT scan showed esophageal stricture with a possibility of malignancy. Repeated multiple biopsies performed 
showed only inflammatory exudate. However, in view of strong clinical suspicion of carcinoma, patient underwent esophagectomy with partial gastrectomy. We received an esophago-gastrectomy specimen, measuring $6.5 \mathrm{~cm}$. The wall of esophagus was externally thickened and nodular, while the cut section showed a stricture with focal mucosal ulceration. No definite mass lesion was identified, either in esophagus or stomach. Routinely stained (H \& E) sections (figure 1: a-c) showed ulcerated oesophageal mucosa with fibro-collagenous tissue in the wall, and multiple granulomas with giant cells and mixed inflammation, seen in muscularis propria and adventitia. Hence special stains were asked, of which Acid fast Bacilli (AFB) was negative and Gomori's Methenamine Silver (GMS) stain showed acutely branched, focally septate fungal hyphae resembling Aspergillus. Multiple sections from esophagus and stomach showed no evidence of malignancy. Hence a diagnosis of "Esophageal granulomatous inflammation with stricture formation due to invasive Aspergillosis" was rendered. A culture was advised which subsequently came positive. Patient had indolent course postoperatively and was started on antifungal to which he responded well.

Case 2: A 60-year-old man presented in emergency with acute abdominal pain with the features of perforative peritonitis. He was taken for emergency exploratory laparotomy, and a distal gastrectomy was done for gastric perforation with an intraoperative suspicion of malignancy. No significant past history could be elicited. Specimen received showed multiple large perforations, lined with black necrotic material, associated with an indurated and flattened mucosa with diffuse thickening of the stomach wall, resembling ? Linitis plastica (figure 2: a). H\&E stained sections showed mucosal ulceration with inflammatory infiltrate associated with necrosis of mucosa and markedly congested blood vessels, few with fresh fibrin thrombi, suggesting ischemic changes (figure 2: b). However, also seen amidst inflammation on closure view were, scattered filamentous structures resembling fungal hyphae positive on GMS stain, which were broad, aseptate with wide angle branching, thus favouring zygomycosis (figure 2: c). Few vessels also showed invasion by these hyphae with thrombus formation (figure 2: d). Thus a histological diagnosis of "Gastric ulcer with perforation due to fungal etiology, most likely mucor" was made, and an advice for a culture was given. Patient subsequently received antifungal treatment, but left against medical advice and was lost for any further follow up.

Case 3: A 32year old man presented with intestinal obstruction with complaints of distension of abdomen with colicky pain, multiple episodes of vomiting and constipation since last three days. He was a known alcoholic and smoker, negative for HIV, but with a past history of Anti-Koch's Treatment (AKT) taken irregularly just for 3 months due to a clinical suspicion of pulmonary and abdominal tuberculosis. On physical examination, there were multiple bilateral posterior triangle Lymph Nodes, largest measuring $1.5 \mathrm{~cm}$, firm, mobile, non-tender. On per abdomen examination, abdomen was distended, tense with palpable bowel loops. His X-ray showed multiple air fluid levels in abdomen, and mass in caecum on CT scan with a possibility of malignancy. Thus, a cervical lymph node FNAC was advised to rule out metastasis. Subsequently, quarter colectomy was performed, and specimen was received in segments, ileum measuring approximately $15 \mathrm{cms}$ and ileo-caecal junction with ascending colon measuring $19 \mathrm{cms}$, with no tubercles or strictures on external examination. On cutting open, a nodular, and soft to firm mass measuring $1 \mathrm{~cm}$ in diameter was seen in ileum, $2 \mathrm{~cm}$ away from the resection margin, which showed greyish white, flashy, glistening cut surface and an adjacent perforated ulcer (figure 3: a, b). The caecum and appendix showed diffuse thickening and a similar cut surface on cutting open with necrotic material in Appendicular lumen (figure 3: c). Few enlarged mesenteric lymph nodes were also found with no caseation. Meanwhile, the FNAC report received showed, necrotizing granulomatous inflammation rich in histiocytes, with intra \& extracellular yeast forms with single eccentric nucleus and surrounding "halo," thus favouring Histoplasmosis. (figure 3: d). Histological sections from Ileum, caecum, appendix and mesenteric nodes also showed multiple granulomas with giant cells and histiocytes having foamy cytoplasm with numerous intracellular tiny dots like organisms, positive on GMS and PAS stain (figure 3:e-f), with narrow based budding. Thus a final diagnosis of "Disseminated Histoplasmosis with gastrointestinal perforation" was made.

\section{Discussion}

In this study, we came across a series of three cases of fungal infections of different species, involving different GI sites, but all presented with a clinical suspicion of a malignancy on $\mathrm{CT}$ / intraoperatively/ on gross pathology. First case was angio-invasive aspergillosis involving esophagus, second was zygomycosis involving stomach, while third was a case of disseminated histoplasmosis involving ileum and caecum.

The importance of fungal infections of the GI tract has increased in parallel with the numbers of patients with organ transplants, AIDS, and other immunodeficiency states. Although fungal infections of the GI tract are often a part of a disseminated disease process, GI symptoms and signs may be the only presenting manifestations of disease. ${ }^{[7]}$ 


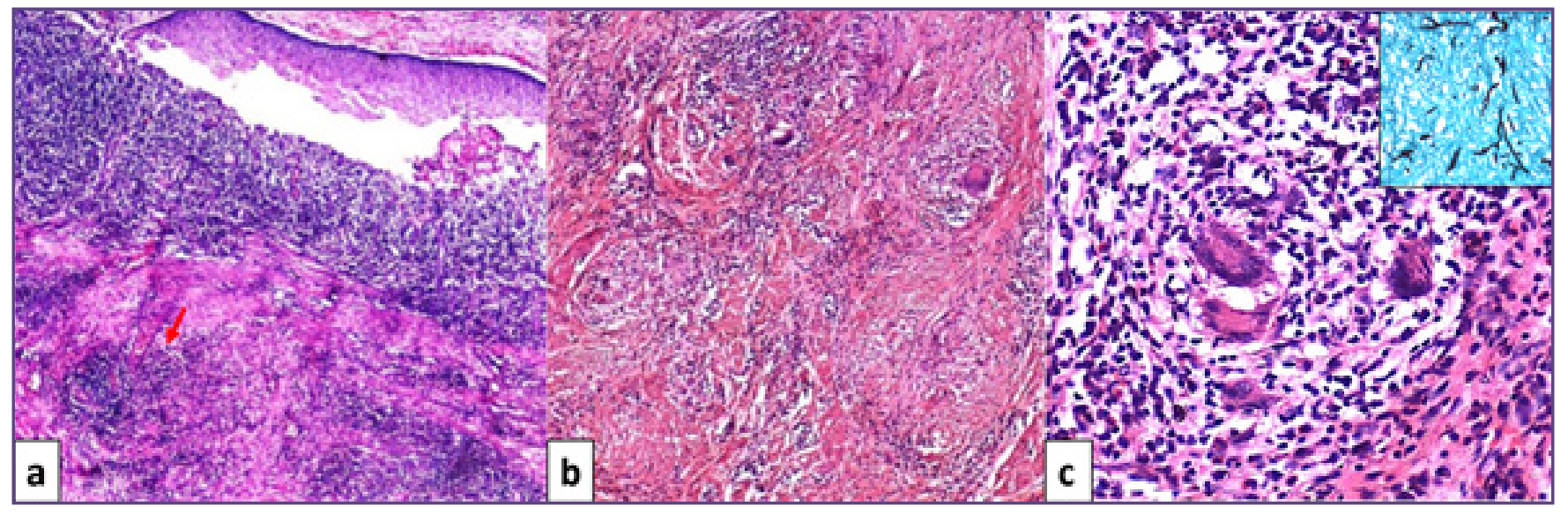

Fig. 1: a) Ulcerated esopghageal mucosa (black arrow), dense inflammatory infiltrate, fibrocollagenous tissue and multiple granulomas (red arrow) in submucosa (H\&E x 40). b) Muscularis propria showing granulomas with giant cells (H\&E x 100) c) Granuloma with giant cells (H\&E x 400) and slender fungal hyphae with acute angle branching on GMS stain (inset).

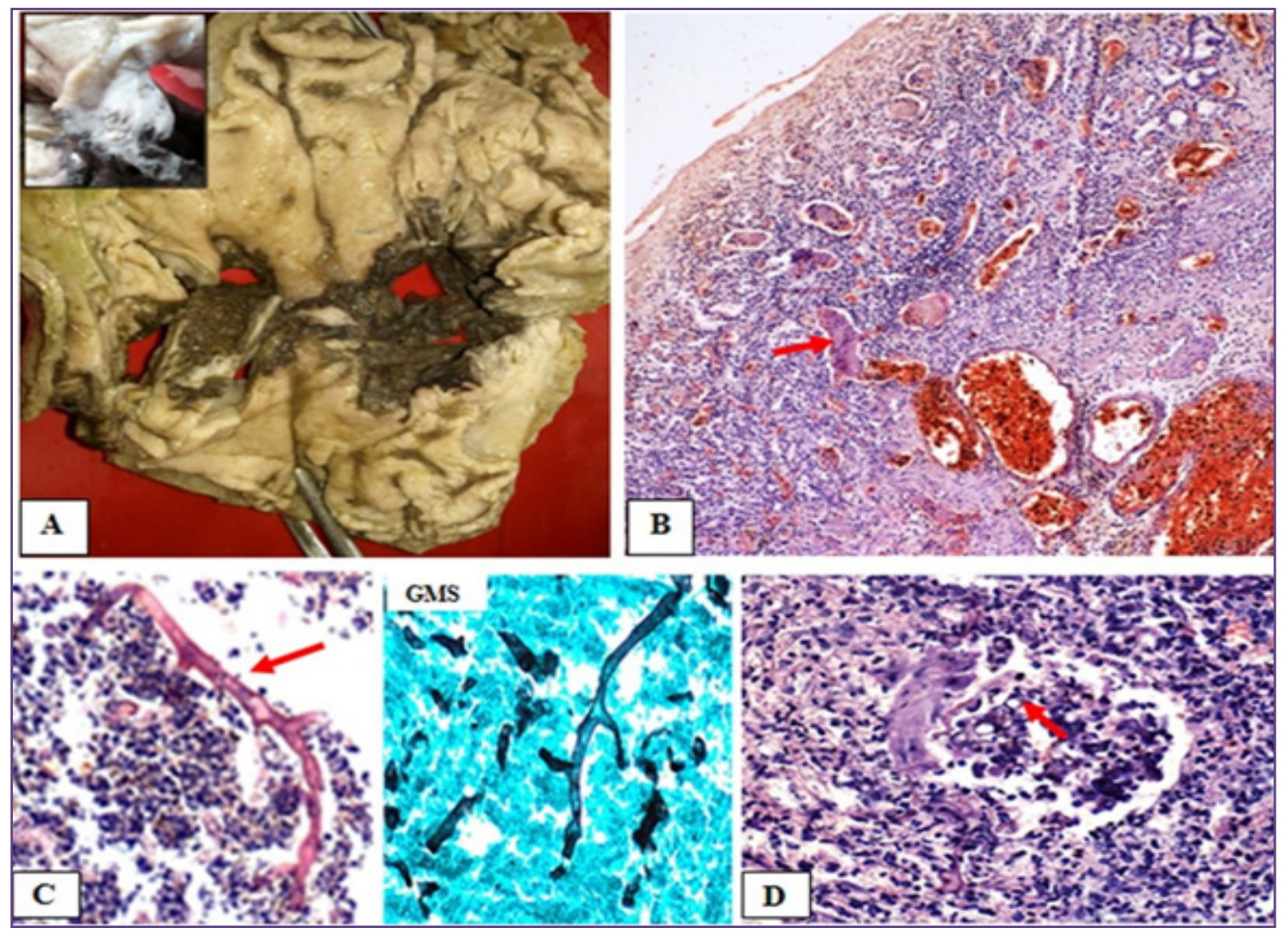

Fig. 2: a) Stomach showing Perforations, lined with black necrotic material, with diffuse thickening of the wall (inset). b) Ulcerated stomach mucosa, inflammatory granulation tissue, congested blood vessels; many with fibrin thrombi (arrow) (H\&E x 40). c) Necro-inflammatory background with broad, pauci-septate fungal hyphae with wide angle branching (H\&E $x$ 400), positive on GMS. d) Angioinvasion, with destruction of arteriolar wall by fungal hyphae (arrow) (H\&E x 400). 


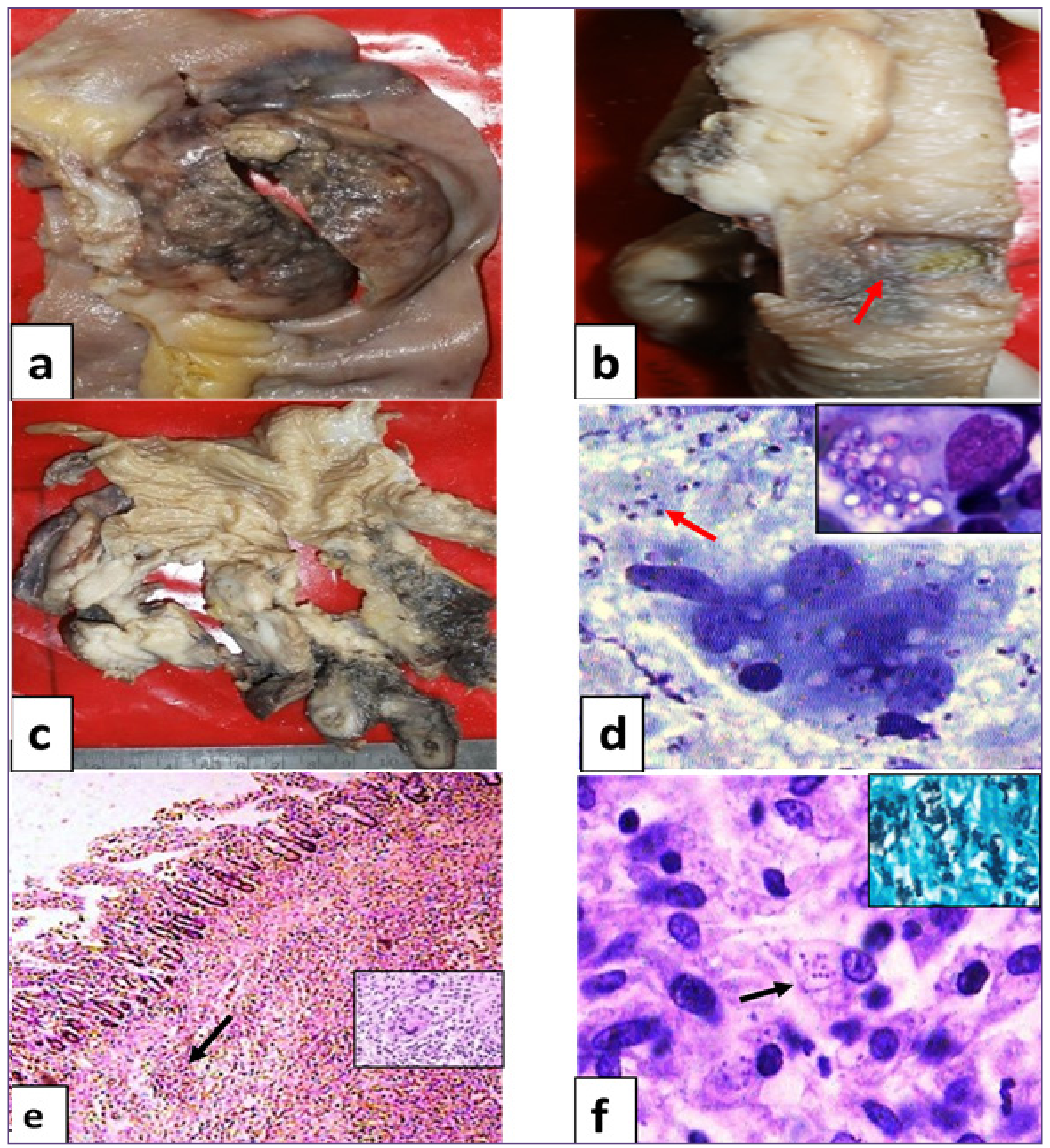

Fig. 3: a) External surface of ileum showing a nodular mass. b) Greyish white, flashy, glistening cut surface with an adjacent ulcer (arrow). c) Diffusely thickened caecum and appendix. d) Cervical lymph node FNAC showing granulomatous inflammation with extracellular (arrow) and intracellular (inset) yeast forms with eccentric nucleus and surrounding "halo" (MGG x 400) e) Ileum showing lymphohistiocytic inflammation with dot like organisms (arrow) (H\&E x 40) and occasional granulomas (inset). f) Histiocytes showing intracellular tiny organisms (arrow) (H\&E x400), positive on GMS stain (inset), with narrow based budding. 
Invasive Aspergillosis (IA) typically involves the lungs, but may also infect the nasal sinuses, central nervous system, and rarely gastrointestinal (GI) system. It can be seen, both in immunocompromised and immunocompetent hosts. Mishra et al ${ }^{[2]}$ and cha et al ${ }^{[8]}$ have both reported GIA in immunocompetent patient. Isolated GIA is very rare (approximately 5\%) even in immunocompromised hosts. Eggiman et al, in a large study of GIA $(\mathrm{n}=1538)$, observed most cases in disseminated disease; isolated gut aspergillosis was very exceptional $(0.8 \%)$. ${ }^{[9]}$ In our study, the patient of GIA was though diabetic, but he did not show signs of any other organ involvement, thus possibly had isolated GI involvement.

The common presenting features of GIA are abdominal pain, fever, weight loss and perforation, and sometimes can even mimic malignancy similar to our case, which is similarly also reported by Mishra $\mathrm{P}$ et al in a case of gastric aspergillosis. ${ }^{[2]}$ Most of the studies done on GIA, have shown lower GI tract as most common site involved (small bowel, colon). However, in certain common pathologic conditions like gastric ulcers and severe gastritis, the mucosal barrier can be penetrated by aspergillus spores and can lead to invasive gastric or upper GIA. ${ }^{[8]}$ Kazan et al. in a review of 21 cases reported most cases involving lower GI tract, and only three from upper GI tract. ${ }^{[10]}$ Further, the esophageal involvement by aspergillosis is uncommon as compared to candidiasis, and odynophagia and dysphagia are usual presenting complaints ${ }^{[3]}$ as seen in our patient too. The clinical manifestations at other sites are often nonspecific, such as abdominal pain, diarrhea, hemorrhage, and occasionally intestinal obstruction and perforation. Stricture however is not a common feature, ${ }^{[8]}$ but was present in our case. The histological features of GIA are same as described at any other site, sometimes with a characteristic nodular infarction like lesion which was not present in our case. The inflammatory response ranges from minimal to marked, with a prominent neutrophilic infiltrate. However, a granulomatous response is not very common, but was quite pronounced in our case. ${ }^{[3]}$

Zygomycetes are filamentous fungi having low intrinsic pathogenicity. However, these fungi can produce fulminant infection in patients with underlying immune compromised conditions. Disease can be disseminated or localized to the rhinocerebral area, lungs, gastrointestinal tract or skin [11], and rarely may it affect healthy individuals; as is reported by Sunitha et al. ${ }^{[4]}$ In our case too, there was no history suggesting any associated immunosuppression. Stomach is the most common site involved in GIZ, accompanied by a very high mortality rate. Other sites being colon followed by ileum. ${ }^{[4]}$ The common clinical presentation of GIZ is massive gastric bleed, associated with non- specific symptoms like nausea, vomiting, abdominal pain or distension, depending upon the site of involvement. ${ }^{[12]}$ Thus, sometimes the presentation can mimic inflammatory bowel disease as reported by Sunitha et al ${ }^{[4]}$ and even malignancy as reported by Debata et al. ${ }^{[13]}$ Our patient also had gastric involvement with a clinical suspicion of malignancy at the time of surgery. On gross pathological examination, the ulcers are the most common manifestation, often large, with rolled and irregular edges that may mimic malignancy as was suspected in our case too. Disseminated zygomycosis rapidly invades vessels resulting in thrombus formation, with infarction, necrosis, hemorrhage, and perforative peritonitis, which can sometimes mimic a primary ischemic pathology especially in older patients ${ }^{[14]}$ as was seen in our case. There could be spread to multiple organs, most frequently lung, however in our case, there was no evidence suggesting any other organ involvement. [15] The gold standard for diagnosis of zygomycosis is demonstration of tissue invasion by fungal hyphae with a classical morphology in histopathological specimen, which was present in our case. Serologic tests presently have no significant role in diagnosis Zygomycosis and positive fungal cultures are seen in only $50 \%$ cases. Polymerase chain reaction can be useful for the diagnosis and for species identification, however is not completely reliable for confirmation of diagnosis. ${ }^{[12]}$ In our case, though culture was advised but patient lost for further follow up.

Histoplasmosis is a systemic fungal infection caused by Histoplasma capsulatum which occurs endemically in some parts of the world but is uncommon in India, with only sporadic reports from different parts of country. ${ }^{[16]}$ Disseminated Histoplasmosis (DH) is an extremely rare mycotic infection, usually occurs secondary to high inoculum infections or direct invasion due to trauma. ${ }^{[17]}$ About $40 \%$ of patients do not have any obvious risk factors. In our case too, there were no findings suggesting any definite immunosuppression, except a previous history of irregular AKT treatment. Doleschal et al reported first case in a immunocompetent patient in Austria from a non-endemic area ${ }^{[18]}$ From India, Bauddha et al have also reported a case of progressive $\mathrm{DH}$ in an immunocompetent male from non-endemic area.${ }^{[19]}$ However, Subramanian et al, in an Indian study analysed 19 cases of DH (10 year period) with three cases of GI involvement and found diabetes mellitus and HIV infection to be most common co-morbid conditions. ${ }^{[20]}$

In clear contrast to the initial presentation of acute histoplasmosis, which almost exclusively involves the respiratory tract, DH most prominently affects the gastrointestinal tract with the typical sites of involvement being terminal ileum and caecum, as seen in our case. 
Majority of the patients have a subclinical disease course ${ }^{[18]}$, and are usually asymptomatic. However in symptomatic patients, the most common presentation is fever, bloody diarrhea, abdominal pain, hepatosplenomegaly and lymphadenopathy. ${ }^{[5,20]}$ In our case too, abdominal pain, lymphadenopathy and possibly hepato-splenomegaly (abdominal distension) were present. However our patient primarily presented with obstructive features (vomiting, constipation). The clinical presentation including endoscopy and radiology sometime can be mistaken for infections like mycobacteria, Entamoeba histolytica; or inflammatory bowel disease, appendicitis and even malignancy. ${ }^{[17,18]}$ In our case too, as patients had obstructive features associated with a caecal mass on CT, hence clinically, a possibility of malignancy was suggested. Sehgal et al have also reported a case of GIH, who was suspected to have colonic cancer in view of the findings seen on scopy and radiology. ${ }^{[16]}$ Microscopic findings usually seen in GIH are diffuse, often nodular lymphohistiocytic infiltrates, showing fungal elements within the cytoplasm of macrophages are pathognomonic of histoplasmosis, while well-formed granulomas are rare. ${ }^{[18]}$ In our case however, multiple well formed granulomas were seen.

In tuberculosis-endemic regions as in the Indian subcontinent, DH can easily be mistaken for tuberculosis owing to its similar clinical presentation like prolonged fever, hepatosplenomegaly, lymphadenopathy; and histoplasmosis must always be considered in the differential diagnosis, particularly if there is no response to empirical anti-tubercular therapy (ATT). ${ }^{[21]}$ The Chest X ray findings in $\mathrm{DH}$ can also mimic $\mathrm{TB}$ as both can show cavitatory lesions and fibrosis. ${ }^{[19]}$ Histology can also be confusing sometimes due to presence of granulomas, and even caseous necrosis Hence it becomes imperative to use appropriate staining and culture techniques to identify this organism especially in the immunocompromised. ${ }^{[20]}$ In our case too, though there was a possible past history with a clinical suspicion of TB, which however could not be confirmed, neither on FNAC (which rather showed histoplasmosis), nor on culture as that was also not done. Though the patient took irregular AKT treatment for a short period but stopped; either due to poor compliance or due to a possible lack of response due to him being a case of histoplasmosis.

\section{Conclusion}

GI fungal infections are uncommon, but not rare. They often get missed due to their overlapping clinical features with various other conditions. Fungal etiology can often be mistaken for ischemic pathology owing to angio-invasion and thrombosis; and also sometimes for malignancy leading to extensive surgeries. Hence, a high index of suspicion is most essential, both by clinician and pathologist to make a correct diagnosis. Therefore, a fungal etiology should always be suspected in every case of a GI ulcer or perforation, and pathologist should always ask relevant special stains. Moreover, a simple, but timely treatment with appropriate antifungal therapy is very crucial as the mortality is still high with certain fungi, especially in patients with immunosuppression, including old age and diabetes.

\section{Acknowledgements}

Dr. Avinash Supe, Professor, GI Surgery, Seth GSMC \& KEM Hospital, Mumbai

Dr. Shilpa Rao, Professor, Gen. Surgery, Seth GSMC \& KEM Hospital, Mumbai

Dr. S.A. Rege, Professor, Gen. Surgery, Seth GSMC \& KEM Hospital, Mumbai

\section{Funding}

Nil

\section{Competing Interests}

Nil

\section{References}

1. Lamps LW, Lai KKT, Milner DA. Fungal Infections of the Gastrointestinal Tract in the Immuno-compromised HostAn Update. Adv Anat Pathol 2014; 21: 217-227

2. Mishra P, Gupta D, Mishra S, Srinivas V. Aspergillosis of Gastrointestinal Tract Mimicking Malignancy: A Case Report. J Clin Exp Pathol 2017;7:1-3

3. Odze RD, Goldblum JR. Surgical pathology of the GI tract, liver, biliary tract, and pancreas. Infect Dis GI Tract 2009; 51-79

4. Shankaralingappa S. Unsuspected invasive gastrointestinal mucormycosis masquerading as inflammatory bowel disease: A pathologist's perspective. Indian J Pathol Microbiol 2019; 62:332-4

5. Nakshabendi R, Berry AC, Miranda D, LaBarbera FD, Kanar O, Nakshabandi A, Nakshabendi I. Primary Histoplasma capsulatum Enterocolitis Mimicking Peptic and Inflammatory Bowel Disease. Case Reports in Gastrointestinal Medicine 2016; 1-3

6. Praneenararat S. Fungal infection of the colon. Clinical and Experimental Gastroenterology 2014:7 415-426

7. Lamps LW. Infectious Disorders of the GI Tract. In: Surgical Pathology of the Gastrointestinal Tract, Liver, Biliary Tract and Pancreas 2009; p.51-79

8. Cha SA, Kim MH, Lim TS, Kim HH, Chang KY, Park HS et al. Invasive Primary Colonic Aspergillosis in the Immunocompetent Host without Classical Risk Factors. Yonsei Med J 2015;56:1453-56 
9. Eggimann P, Chevrolet JC, Starobinski M, Majno P, Totsch M, B. Chapuis B. et al. Primary invasive aspergillosis of the digestive tract: report of two cases and review of the literature. Infection 2006; 34: 333-38

10. Kazan E, Maertens J, Herbrecht R, Weisser M, Gachot B, Vekhoff A et al. A retrospective series of gut aspergillosis in haematology patients. Clin Microbiol Infect 2011; 17: 588-94

11. Patra S, Vij M, Chirla DK, Kumar N, Samal SC. Unsuspected invasive neonatal gastrointestinal mucormycosis: A clinicopathological study of six cases from a tertiary care hospital. J Indian Assoc Pediatr Surg 2012; 17:153-6

12. Sruthi J, Shrivalli N, Aruna Raman P and Ashitha S. Colonic Angioinvasive Mucormycosis - Case Report. Austin J Radiol 2019; 6: 1094

13. Debata et al. An unusual presentation of colonic mucormycosis mimicking carcinoma colon- a surgeon's perspective International Journal of Surgery Case Reports 2015; 10: 248-51

14. Lee ZJ, Chia C, Busmani I, Wong WK. A rare cause of ischemic gut: A case report. International Journal of Surgery Case reports 2016; 20:114-17

15. Choi WT, Chang TT, Gill RM. Gastrointestinal Zygomycosis Masquerading as Acute Appendicitis. Case
Rep Gastroenterol 2016;10:81-87

16. Sehgal S, Chawla R, Loomba PS, Mishra B. Gastrointestinal Histoplasmosis presenting as colonic pseudotumour. Indian journal of Medical Microbiology 2007; 26: 187-189.

17. Romano RC, Soape MM, Thirumala S, Ghandour E. Disseminated histoplasmosis mimicking metastatic disease of the colon and omentum: Report of a case and literature review. Arab Journal of Gastroenterology 2015; 16 : 66-68

18. Doleschal B, Rodhammer T, Tsybrovskyy O, Achberger KJ, Lang F. Disseminated Histoplasmosis: A Challenging Differential Diagnostic Consideration for Suspected Malignant Lesions in the Digestive Tract. Case Rep Gastroenterol 2016;10:653-60.

19. Bauddha NK, Jadon RS, Mondal S, Vikram NK, Sood R. Progressive disseminated histoplasmosis in an immunocompetent adult: A case report Intractable \& Rare Diseases Research 2018; 7:126-129.

20. Subramanian S, Abraham OC, Rupali P, Zachariah A, Mathews MS, Mathai D. Disseminated histoplasmosis. J Assoc Physicians India. 2005;53:185-9

21. Randhawa HS, Gugnani HC. Occurence of Histoplasmosis in the Indian Sub-Continent: An Overview and Update. J Med Res Prac 2018; 7: 71-83.

*Corresponding author:

Chaturvedi Rachana A, Zeon B 403, Ajmera I-Land, Bhaktipark, Wadala East, Mumbai.

Phone: +91 9967017267

Email: rachanachaturvedi@yahoo.co.in

Date of Submission $\quad: 09 / 06 / 2021$

Date of Final Revision : 13/08/2021

Date of Acceptance $\quad: 16 / 08 / 2021$

Financial or other Competing Interests: None.

Date of Publication : :30/08/2021 